\title{
Doping and temperature dependence of electronic Raman response in cuprate superconductors
}

\author{
Zhihao Geng and Shiping Feng \\ Department of Physics, Beijing Normal University, Beijing 100875, China
}

\begin{abstract}
The doping and temperature dependence of the electronic Raman response in cuprate superconductors is studied within the kinetic energy driven superconducting mechanism. It is shown that the temperature dependent depletion at low-energy shifts is faster in the $B_{1 g}$ symmetry than in the $B_{2 g}$ symmetry. In analogy to the domelike shape of the doping dependent superconducting transition temperature, the maximal peak energy in the $B_{2 g}$ channel occurs around the optimal doping, and then decreases in both underdoped and overdoped regimes. Moreover, the overall density of Cooper pairs increases with increasing doping in the underdoped regime.
\end{abstract}

Cuprate superconductors are doped Mott insulators with the strong electron correlation dominating the entire phase diagram [1]. After over 20 years extensive studies, it has become clear that superconductivity in doped cuprates results when electrons pair up into Cooper pairs [2] as in the conventional superconductors [3]. However, the superconducting (SC) transition temperature $T_{c}$ is strongly dependent on doping, and takes a domelike shape with the underdoped and overdoped regimes on each side of the optimal doping where $T_{c}$ researches its maximum. Moreover, as a natural consequence of the unconventional SC mechanism [4], the Cooper pairs have a dominant d-wave symmetry [1, 2]. Since many physical properties of the two-particle electron dynamics have been attributed to particular characteristics of low energy excitations determined by the electronic Raman response (ERR) [5, 6], in this case, a central issue to clarify the nature of the electron dynamics is how ERR evolves with doping and temperature.

Experimentally, by virtue of systematic studies using the ERR measurement technique, some essential features of the evolution of the two-particle excitations in cuprate superconductors with doping and temperature in the SC state have been established [5-16]: (a) the $B_{1 g}$ orientation projects out excitations around the antinodal region in the Brillouin zone where the energy gap is maximal; (b) the $B_{2 g}$ orientation projects out excitations around the nodal region where the energy gap vanishes; (c) as a consequence of both cases (a) and (b), ERR has a peak at the antinodes in the $B_{1 g}$ symmetry and at slightly lower energy at the nodes in the $B_{2 g}$ symmetry; however, (d) in the underdoped regime, the peak in the $B_{1 g}$ symmetry shifts to a higher energy with decreasing doping and tracks of the antinodal gap, while the peak in the $B_{2 g}$ symmetry shows the same doping dependence as $T_{c}$ and shifts to a lower energy with decreasing doping; and (e) the $B_{2 g}$ response depends linearly on energy $\omega$ in the limit $\omega \sim 0$ for the energy gap vanishing along with the diagonal directions of the Brillouin zone, while for the $B_{1 g}$ orientation, the Raman vertex vanishes along with the energy gap at the same directions, which yields an additional $\omega^{2}$ contribution from the line nodes of the vertex, and the resulting ERR varies as $\omega^{3}$. Theoretically, an agreement has emerged that a simple d-wave
Bardeen-Cooper-Schrieffer (BCS) formalism is useful in the phenomenological description of ERR in cuprate superconductors [6, 17 21]. In particular, incorporating the d-wave BCS formalism with the spin-fluctuation model 19 21], the role of the collective modes in the ERR experiment on cuprate superconductors is studied [20], and the result shows that the collective modes of the amplitude fluctuation of the d-wave gap yield a broad peak above the threshold in the $B_{1 g}$ spectrum. Moreover, it has been shown that ERR in the $B_{1 g}$ symmetry allows one to distinguish between phonon-mediated and magnetically mediated d-wave superconductivity [21]. However, to the best of our knowledge, ERR in cuprate superconductors has not been treated starting from a microscopic SC theory, and no explicit calculation of the doping dependence of ERR has been made so far. Very recently, using a simple relationship between the integrated ERR and density of Cooper pairs (DOCP), it is shown experimentally [22] that in a similar way to the superfluid density [23], the overall DOCP strongly increases with increasing doping in the underdoped regime 22]. Thus this recently discovered doping dependence of the overall DOCP is also calling for an explanation.

In this Letter, we study ERR in cuprate superconductors based on the kinetic energy (KE) driven SC mechanism [24]. We evaluate explicitly the ERR function in terms of the Raman density-density correlation function (RDDCF), and qualitatively reproduce some main features of ERR in cuprate superconductors $7-16$, 22]. In particular, we show that the temperature dependent depletion at low-energy shifts is faster in the $B_{1 g}$ symmetry than in the $B_{2 g}$ symmetry. Moreover, in analogy to the domelike shape of the doping dependence of $T_{c}$, the maximal peak energy in the $B_{2 g}$ channel occurs around the optimal doping, and then decreases in both underdoped and overdoped regimes. Furthermore, the overall DOCP increases with increasing doping in the underdoped regime.

We start from the $t$ - $J$ model [1, 4],

$$
\begin{aligned}
H & =-t \sum_{i \hat{\eta} \sigma} C_{i \sigma}^{\dagger} C_{i+\hat{\eta} \sigma}+t^{\prime} \sum_{i \hat{\eta}^{\prime} \sigma} C_{i \sigma}^{\dagger} C_{i+\hat{\eta}^{\prime} \sigma} \\
& +\mu \sum_{i \sigma} C_{i \sigma}^{\dagger} C_{i \sigma}+J \sum_{i \hat{\eta}} S_{i} \cdot S_{i+\hat{\eta}},
\end{aligned}
$$

acting on the Hilbert subspace with no doubly occupied 
site, i.e., $\sum_{\sigma} C_{i \sigma}^{\dagger} C_{i \sigma} \leq 1$, where $\hat{\eta}= \pm \hat{x}, \pm \hat{y}, \hat{\eta}^{\prime}= \pm \hat{x} \pm \hat{y}$, $C_{i \sigma}^{\dagger}\left(C_{i \sigma}\right)$ is the creation (annihilation) operator of an electron with spin $\sigma, \mathbf{S}_{i}=\left(S_{i}^{x}, S_{i}^{y}, S_{i}^{z}\right)$ are spin operators, and $\mu$ is the chemical potential. To deal with the constraint of no double occupancy, the charge-spin separation (CSS) fermion-spin theory [25, 26] has been developed, where the physics of no double occupancy is taken into account by representing the constrained electron operators as $C_{i \uparrow}=h_{i \uparrow}^{\dagger} S_{i}^{-}$and $C_{i \downarrow}=h_{i \downarrow}^{\dagger} S_{i}^{+}$, with the spinful fermion operator $h_{i \sigma}=e^{-i \Phi_{i \sigma}} h_{i}$ that describes the charge degree of freedom together with some effects of spin configuration rearrangements due to the presence of the doped hole itself, while the spin operator $S_{i}$ represents the spin degree of freedom, then the electron local constraint for the single occupancy is satisfied in analytical calculations. In this CSS fermion-spin representation, the $t-J$ model (11) is expressed as,

$$
\begin{aligned}
H & =t \sum_{i \hat{\eta}}\left(h_{i+\hat{\eta} \uparrow}^{\dagger} h_{i \uparrow} S_{i}^{+} S_{i+\hat{\eta}}^{-}+h_{i+\hat{\eta}_{\downarrow}}^{\dagger} h_{i \downarrow} S_{i}^{-} S_{i+\hat{\eta}}^{+}\right) \\
& -t^{\prime} \sum_{i \hat{\eta}^{\prime}}\left(h_{i+\hat{\eta}^{\prime} \uparrow}^{\dagger} h_{i \uparrow} S_{i}^{+} S_{i+\hat{\eta}^{\prime}}^{-}+h_{i+\hat{\eta}^{\prime} \downarrow}^{\dagger} h_{i \downarrow} S_{i}^{-} S_{i+\hat{\eta}^{\prime}}^{+}\right) \\
& -\mu \sum_{i \sigma} h_{i \sigma}^{\dagger} h_{i \sigma}+J_{\mathrm{eff}} \sum_{i \hat{\eta}} S_{i} \cdot S_{i+\hat{\eta}},
\end{aligned}
$$

with $J_{\text {eff }}=(1-\delta)^{2} J$, and $\delta=\left\langle h_{i \sigma}^{\dagger} h_{i \sigma}\right\rangle=\left\langle h_{i}^{\dagger} h_{i}\right\rangle$ is the hole doping concentration.

For an understanding of the physical properties of cuprate superconductors in the SC state, the KE driven $\mathrm{SC}$ mechanism has been developed [24] based on the CSS fermion-spin theory, where the interaction between charge carriers and spins from the KE term in the $t$ $J$ model (2) induces the d-wave charge carrier pairing state by exchanging spin excitations, then the electron Cooper pairs originating from the charge carrier pairing state are due to the charge-spin recombination, and their condensation reveals the SC ground-state. In particular, this $\mathrm{SC}$ state is a conventional BCS-like with the d-wave symmetry [26, 27], so that the basic d-wave BCS formalism is still valid in discussions of the low energy electron quasiparticle excitations of cuprate superconductors, although the pairing mechanism is driven by $\mathrm{KE}$ by exchanging spin excitations. Following these previous discussions [24, 27], the full charge carrier Green function can be obtained in the Nambu representation as,

$$
g\left(\mathbf{k}, i \omega_{n}\right)=Z_{\mathrm{hF}} \frac{i \omega_{n} \tau_{0}+\bar{\xi}_{\mathbf{k}} \tau_{3}-\bar{\Delta}_{\mathrm{hZ}}(\mathbf{k}) \tau_{1}}{\left(i \omega_{n}\right)^{2}-E_{\mathrm{hk}}^{2}},
$$

where $\tau_{0}$ is the unit matrix, $\tau_{1}$ and $\tau_{3}$ are Pauli matrices, the charge carrier quasiparticle spectrum $E_{h \mathbf{k}}=$ $\sqrt{\bar{\xi}_{\mathbf{k}}^{2}+\left|\bar{\Delta}_{h Z}(\mathbf{k})\right|^{2}}$ with the renormalized d-wave charge carrier pair gap function $\bar{\Delta}_{h Z}(\mathbf{k})=\bar{\Delta}_{h Z}\left[\cos k_{x}-\cos k_{y}\right] / 2$, while the charge carrier quasiparticle coherent weight $Z_{h F}$ and other notations are defined as same as in Ref. [27], and have been determined by the self-consistent calculation [24, 27].

In the CSS fermion-spin representation [25], the electron Green's function,

$$
G\left(\mathbf{k}, i \omega_{n}\right)=\left(\begin{array}{ll}
G_{11}\left(\mathbf{k}, i \omega_{n}\right), & G_{12}\left(\mathbf{k}, i \omega_{n}\right) \\
G_{21}\left(\mathbf{k}, i \omega_{n}\right), & G_{22}\left(\mathbf{k}, i \omega_{n}\right)
\end{array}\right)
$$

is a convolution of the spin Green's function and charge carrier Green's function (3), and its diagonal and offdiagonal components $G_{11}\left(i-j, t-t^{\prime}\right)=\left\langle\left\langle C_{i \sigma}(t) ; C_{j \sigma}^{\dagger}\left(t^{\prime}\right)\right\rangle\right\rangle$ and $G_{21}\left(i-j, t-t^{\prime}\right)=\left\langle\left\langle C_{i \uparrow}^{\dagger}(t) ; C_{j \downarrow}^{\dagger}\left(t^{\prime}\right)\right\rangle\right\rangle$ have been given in Ref. [27].

The ERR function $\tilde{S}(\mathbf{q}, \omega)$ is obtained from the imaginary part of $\operatorname{RDDCF} \tilde{\chi}(\mathbf{q}, \omega)$ as [6],

$$
\tilde{S}(\mathbf{q}, \omega)=-\frac{1}{\pi}\left[1+n_{B}(\omega)\right] \operatorname{Im} \tilde{\chi}(\mathbf{q}, \omega),
$$

where $n_{B}(\omega)$ is the boson distribution function, while $\operatorname{RDDCF} \tilde{\chi}(\mathbf{q}, \omega)$ is defined as,

$$
\left.\tilde{\chi}\left(\mathbf{q}, \tau-\tau^{\prime}\right)=-\left\langle T \rho_{\gamma}(\mathbf{q}, \tau) \rho_{\gamma}\left(-\mathbf{q}, \tau^{\prime}\right)\right]\right\rangle,
$$

where $\rho_{\gamma}(\mathbf{q})=\sum_{\mathbf{k}} \gamma_{\mathbf{k}} C_{\mathbf{k}+\frac{\mathbf{q}}{2}}^{\dagger} \tau_{3} C_{\mathbf{k}-\frac{\mathbf{q}}{2}}$ is the Raman density operator in the Nambu representation, with the bare Raman vertex $\gamma_{\mathbf{k}}$ has been classified by the representations $B_{1 g}, B_{2 g}$, and $A_{1 g}$ of the point group $D_{4 h}$ as [6, 18],

$$
\gamma_{\mathbf{k}}= \begin{cases}b_{\omega_{i}, \omega_{s}}\left[\cos \left(k_{x} a\right)-\cos \left(k_{y} a\right)\right] / 4, & B_{1 g} \\ b_{\omega_{i}, \omega_{s}}^{\prime} \sin \left(k_{x} a\right) \sin \left(k_{y} a\right), & B_{2 g} \\ a_{\omega_{i}, \omega_{s}}\left[\cos \left(k_{x} a\right)+\cos \left(k_{y} a\right)\right] / 4, & A_{1 g}\end{cases}
$$

respectively, where as a qualitative discussion, the magnitude of the energy dependence of the prefactors $b, b^{\prime}$ and $a$ can be rescaled to units. In this case, $\tilde{\chi}(\mathbf{q}, \omega)$ can be obtained in terms of the electron Green's function (4) as,

$$
\begin{aligned}
& \tilde{\chi}_{\gamma_{1} \gamma_{2}}(\mathbf{q}, \omega)=\frac{1}{2} Z_{F}^{2} \frac{1}{N^{3}} \sum_{\mathbf{k}, \mathbf{p}, \mathbf{p}^{\prime}} \gamma_{1 \mathbf{k}} \gamma_{2 \mathbf{k}} \frac{B_{\mathbf{p}} B_{\mathbf{p}^{\prime}}}{\omega_{\mathbf{p}} \omega_{\mathbf{p}^{\prime}}} \sum_{\mu \nu \mu^{\prime} \nu^{\prime}=1,2} L_{\mu}(\mathbf{p}, \mathbf{p}+\mathbf{k}+\mathbf{q}) L_{\mu^{\prime}}\left(\mathbf{p}^{\prime}, \mathbf{p}^{\prime}+\mathbf{k}\right)\left[U_{\nu h \mathbf{p}+\mathbf{k}+\mathbf{q}}^{2} U_{\nu^{\prime} h \mathbf{p}^{\prime}+\mathbf{k}}^{2}\right. \\
& \left.-\quad(-1)^{\nu+\nu^{\prime}} \frac{\bar{\Delta}_{h Z}(\mathbf{p}+\mathbf{k}+\mathbf{q}) \bar{\Delta}_{h Z}\left(\mathbf{p}^{\prime}+\mathbf{k}\right)}{4 E_{h \mathbf{p}+\mathbf{k}+\mathbf{q}} E_{h \mathbf{p}^{\prime}+\mathbf{k}}}\right] \frac{n_{F}\left[(-1)^{\nu^{\prime}} E_{h \mathbf{p}^{\prime}+\mathbf{k}}-(-1)^{\mu^{\prime}+\nu^{\prime}} \omega_{\mathbf{p}^{\prime}}\right]-n_{F}\left[(-1)^{\nu} E_{h \mathbf{p}+\mathbf{k}+\mathbf{q}}-(-1)^{\mu+\nu} \omega_{\mathbf{p}}\right]}{\omega+(-1)^{\nu^{\prime}} E_{h \mathbf{p}^{\prime}+\mathbf{k}}-(-1)^{\mu^{\prime}+\nu^{\prime}} \omega_{\mathbf{p}^{\prime}}-(-1)^{\nu} E_{h \mathbf{p}+\mathbf{k}+\mathbf{q}}-(-1)^{\mu+\nu} \omega_{\mathbf{p}}}
\end{aligned}
$$


where the electron quasiparticle coherent weight $Z_{F}=$ $Z_{h F} / 2, n_{F}(\omega)$ is the fermion distribution function, $U_{1 h \mathbf{k}}^{2}=\left(1+\bar{\xi}_{\mathbf{k}} / E_{h \mathbf{k}}\right) / 2$ and $U_{2 h \mathbf{k}}^{2}=\left(1-\bar{\xi}_{\mathbf{k}} / E_{h \mathbf{k}}\right) / 2$, while the spin excitation spectrum $\omega_{\mathbf{p}}$ and $B_{\mathbf{p}}$ have been given in Ref. [27].

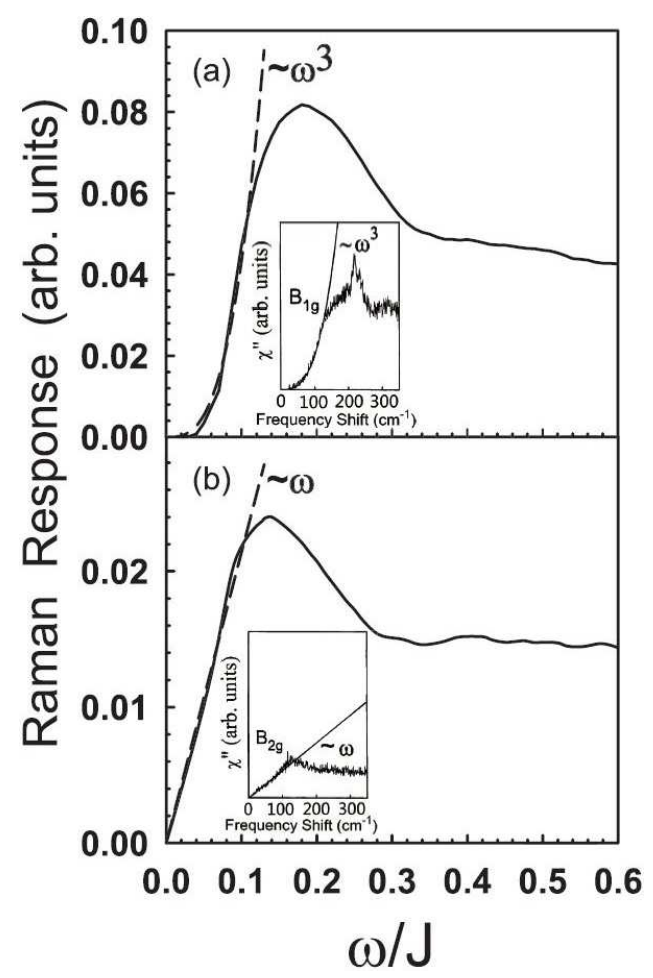

FIG. 1: (a) $B_{1 g}$ and (b) $B_{2 g}$ spectra as a function of energy at $p=0.15$ for $T=0.002 J$. The dashed lines are a cubic and a linear fit for the low-energy $B_{1 g}$ and $B_{2 g}$ spectra, respectively. Inset: the corresponding experimental results taken from Ref. 9.

In cuprate superconductors, although the values of $J$ and $t$ are believed to vary somewhat from compound to compound [1], however, as a qualitative discussion, the commonly used parameters in this paper are chosen as $t / J=2.5$ and $t^{\prime} / t=0.3$. In this case, we have performed a calculation for the ERR function (5) in both $B_{1 g}$ and $B_{2 g}$ orientations, and the results of (a) the $B_{1 g}$ and (b) $B_{2 g}$ spectra with doping $p=0.15$ at temperature $T=0.002 J$ are plotted in Fig. 1. For comparison, the corresponding experimental results [9] are also presented in Fig. 11 (inset). Obviously, both $B_{1 g}$ and $B_{2 g}$ spectra are characterized by the presence of the pairbreaking peaks, however, the peak in the $B_{1 g}$ geometry develops at roughly $30 \%$ higher energy than that in the $B_{2 g}$ geometry. Moreover, we have also fitted our present results, and found that the low-energy spectra almost rise as $\omega^{3}$ in the $B_{1 g}$ channel and linearly with $\omega$ in the $B_{2 g}$ channel. However, in contrast to the conventional superconductors, the intensities of ERR in both $B_{1 g}$ and $B_{2 g}$ channels are equal to zero when energy $\omega=0$, in other words, there are no sharp onset of the intensities at a threshold. This can be understood from the physical property of RDDCF (8). In low temperatures, the spins center around the $[ \pm \pi, \pm \pi]$ points, then the main contribution from the spins comes from the $[ \pm \pi, \pm \pi]$ points. In this case, RDDCF (8) in the momentum transfers $\mathbf{q} \sim 0$ limit can be approximately reduced in terms of $\omega_{\mathbf{p}=[ \pm \pi, \pm \pi]} \sim 0$ and one of the self-consistent equations $1 / 2=\left\langle S_{i}^{+} S_{i}^{-}\right\rangle=(1 / N) \sum_{\mathbf{p}} B_{\mathbf{p}} \operatorname{coth}\left(\beta \omega_{\mathbf{p}} / 2\right) /\left(2 \omega_{\mathbf{p}}\right)$ as [27],

$$
\begin{aligned}
\tilde{\chi}_{\gamma_{1} \gamma_{2}}(\mathbf{q} \sim 0, \omega) & \approx Z_{F}^{2} \frac{1}{N} \sum_{\mathbf{k}} \gamma_{1 \mathbf{k}} \gamma_{2 \mathbf{k}} \frac{\bar{\Delta}_{Z}^{2}(\mathbf{k})}{E_{\mathbf{k}}^{2}} \tanh \left[\frac{1}{2} \beta E_{\mathbf{k}}\right] \\
& \times\left(\frac{1}{2 E_{\mathbf{k}}+\omega}+\frac{1}{2 E_{\mathbf{k}}-\omega}\right),
\end{aligned}
$$

where the renormalized d-wave electron pair gap function $\bar{\Delta}_{Z}(\mathbf{k}) \approx \bar{\Delta}_{h Z}\left(\mathbf{k}+\mathbf{k}_{\mathbf{A}}\right)$ with $\mathbf{k}_{\mathbf{A}}=[\pi, \pi]$, and the electron quasiparticle spectrum $E_{\mathbf{k}} \approx E_{h \mathbf{k}+\mathbf{k}_{\mathbf{A}}}$. Since the $\mathrm{d}$-wave gap function vanishes along the diagonal directions of the Brillouin zone, then as seen from Eq. (9), this leads to an absence of the threshold in cuprate superconductors. These results are qualitatively consistent with the experimental results [7-9].

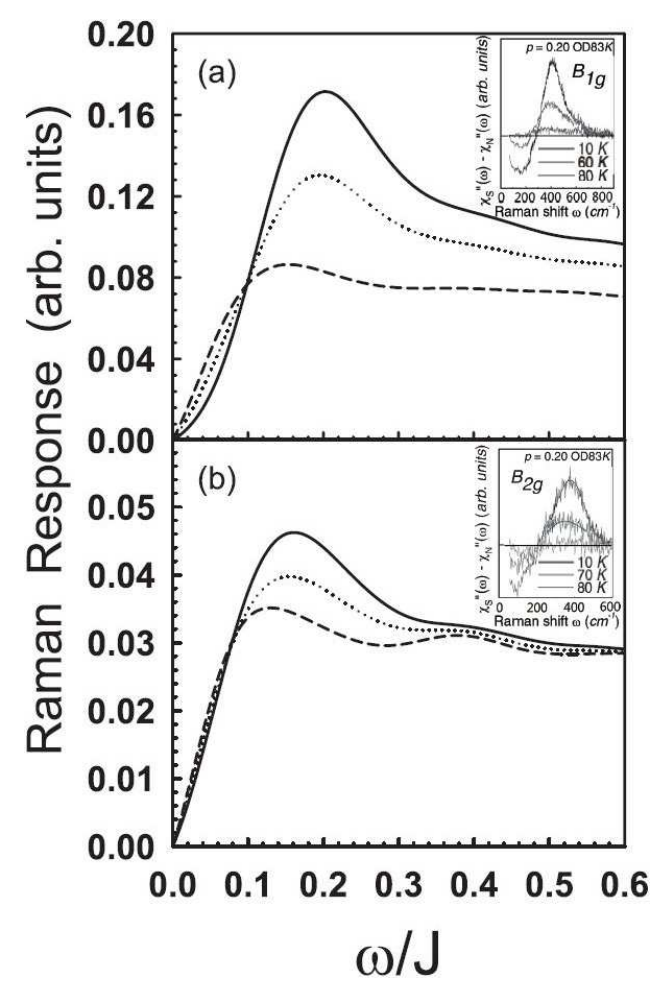

FIG. 2: (a) $B_{1 g}$ and (b) $B_{2 g}$ spectra as a function of energy at $p=0.20$ for $T=0.03 J$ (solid line), $T=0.05 J$ (dotted line), and $T=0.07 J$ (dashed line). Inset: the corresponding experimental results taken from Ref. 16.

For a better understanding of the evolution of ERR with temperature, we have further performed a calculation for the ERR function (5) with different temperatures, and the results of $\tilde{S}(\mathbf{q}, \omega)$ in (a) the $B_{1 g}$ and (b) $B_{2 g}$ channels as a function of energy with $T=0.03 \mathrm{~J}$ 


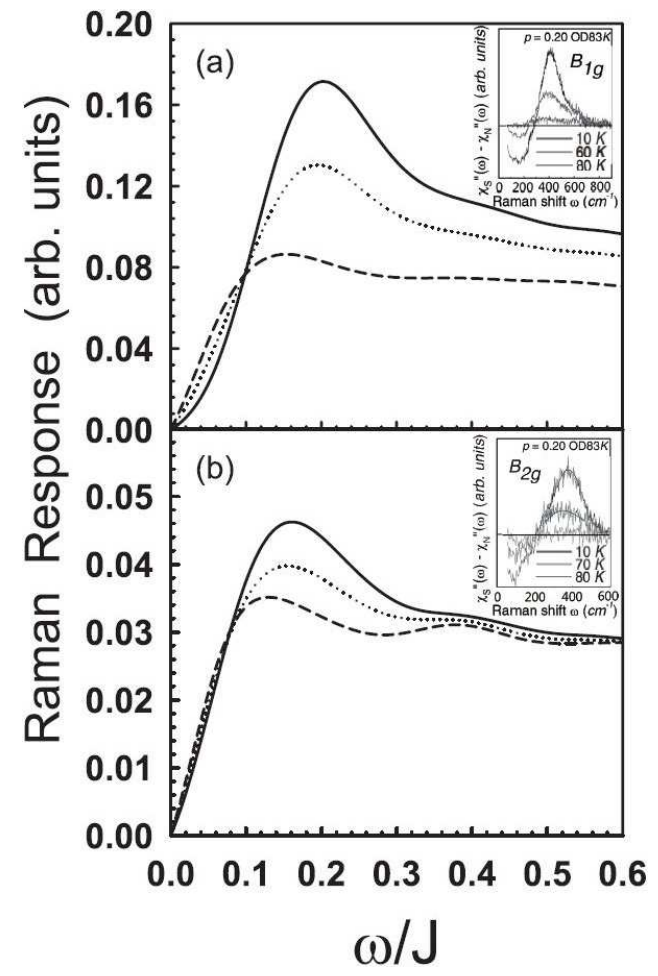

FIG. 3: (a) $B_{1 g}$ and (b) $B_{2 g}$ spectra as a function of energy at $p=0.20$ (solid line) and $p=0.22$ (dotted line) for $T=0.002 J$. Inset: the corresponding experimental results taken from Ref. 12 .

(solid line), $T=0.05 \mathrm{~J}$ (dotted line), and $T=0.07 \mathrm{~J}$ (dashed line) for $p=0.20$ are plotted in Fig. 2 in comparison with the corresponding experimental results [16] (inset). Our results show that there is a strong temperature dependence of the peaks in both $B_{1 g}$ and $B_{2 g}$ orientations in the overdoped regime, where both $B_{1 g}$ and $B_{2 g}$ peaks at low temperatures soften in energy and decreases in intensity as the temperature is raised. Moreover, as seen from Eq. (9), the intensity of the peak follows a pair gap type temperature dependence, and disappears at $T_{c}$. Simultaneously, the low energy continuum grows in agreement with the transfer of spectral weight. Furthermore, the temperature dependent depletion at lowenergy shifts is faster in the $B_{1 g}$ symmetry than in the $B_{2 g}$ symmetry. Although the strong temperature dependence of the $B_{2 g}$ spectrum throughout the SC dome and $B_{1 g}$ spectrum in the overdoped regime are qualitatively consistent with the corresponding experimental results [15, 16], the temperature dependence of the $B_{1 g}$ spectrum in the underdoped regime is in disagreement with the corresponding experimental result [15, 16], where the nontemperature dependence (or weakly increases as the temperature is raised) is observed. This reflects that the excitations observed in the $B_{1 g}$ channel is only related to superconductivity in the overdoped regime, and then ERR in the $B_{1 g}$ and $B_{2 g}$ channels become drastically distinct in the underdoped regime, which can be seen more clearly in the following discussions of the doping depen- dence of ERR in the $B_{1 g}$ channel.

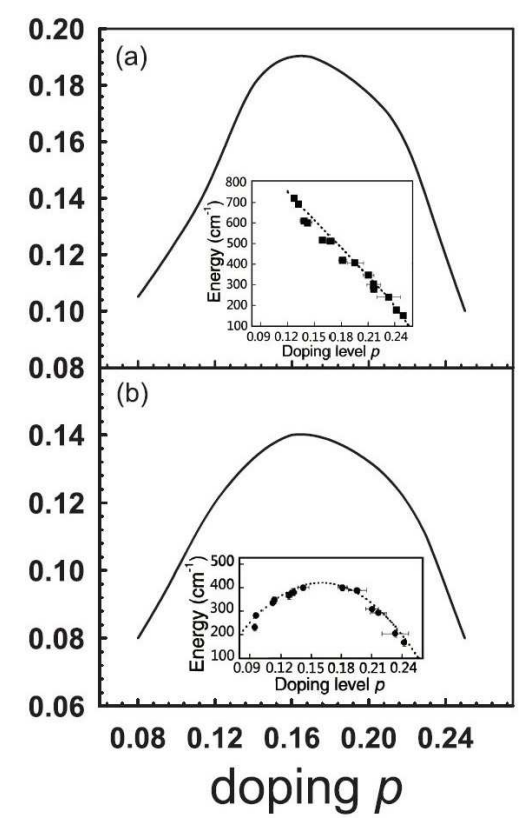

FIG. 4: (a) $B_{1 g}$ and (b) $B_{2 g}$ peaks as a function of doping for $T=0.002 J$. Inset: the corresponding experimental results taken from Ref. 15.

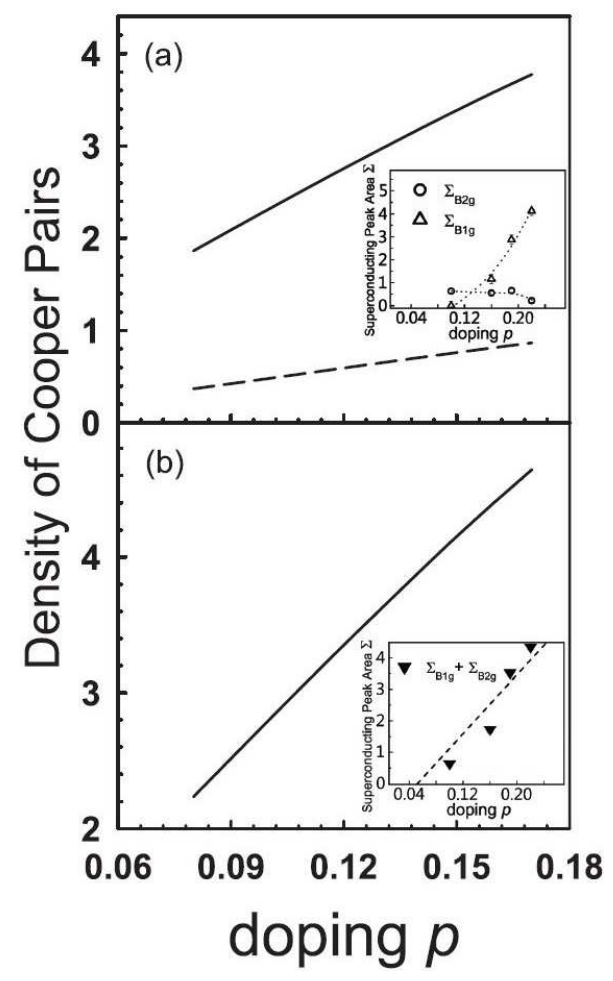

FIG. 5: Doping dependence of (a) the density of Cooper pairs in the $B_{1 g}$ (solid line) and $B_{2 g}$ (dashed line) channels, and (b) the sum of the densities of Cooper pairs in the $B_{1 g}$ and $B_{2 g}$ channels for $T=0.002 J$. Inset: the corresponding experimental results taken from Ref. 22 . 
To analyze the evolution of ERR in Fig. 1 with doping, we have made a series of calculations for the ERR function (5) with different dopings, and the results of (a) the $B_{1 g}$ and (b) $B_{2 g}$ spectra as a function of energy with $p=0.20$ (solid line) and $p=0.22$ (dotted line) at $T=0.002 J$ are plotted in Fig. 3 in comparison with the corresponding experimental results [12] (inset). It is shown clearly that although the intensities of the peaks in both $B_{1 g}$ and $B_{2 g}$ symmetries are strongly suppressed in the overdoped regime when reducing doping, the peak energies change drastically, i.e., the peak energies in both $B_{1 g}$ and $B_{2 g}$ channels decrease with increasing doping. Furthermore, we have found that the peak energies in both $B_{1 g}$ and $B_{2 g}$ channels increase with increasing doping in the underdoped regime. To show this point clearly, we have calculated the ERR function (5) throughout the SC dome, and then employed the shift of the leading-edge mid-point as a measurement of the magnitude of the pair gap at each doping just as it has been done in the ERR experiments [10 15]. The results for the extracted (a) $B_{1 g}$ and (b) $B_{2 g}$ peaks as a function of doping with $T=0.002 J$ are plotted in Fig. 4 For comparison, the corresponding experimental results [15] are also presented in Fig. 44 (inset). The result of the peak energy in the $B_{2 g}$ symmetry continuously follows $T_{c}$ throughout the SC dome as $\omega_{\text {peak }}^{B_{2 g}} \propto T_{c}$, i.e., the maximal peak energy $\omega_{\text {peak }}^{B_{2 g}}$ in the $B_{2 g}$ symmetry occurs around the optimal doping, and then decreases in both underdoped and overdoped regimes. In this sense, it can be identified with the SC gap in the whole doping range, in qualitative agreement with the experimental results [10 15]. However, in the $B_{1 g}$ orientation, although the doping dependence of the peak energy is qualitatively consistent with the experimental data in the overdoped regime, it is in disagreement with the experimental data in the underdoped regime [10 15], where the peak energy increases essentially linearly as doping is reduced. This reflects that the peak energy in the $B_{1 g}$ symmetry is progressively disconnected from superconductivity as one goes from the overdoped regime to the underdoped regime.

Now we turn to discuss the evolution of DOCP with doping. In the case of the low temperatures and momentum transfers $\mathbf{q} \sim 0$ limit, the integral of the imaginary part of RDDCF over energy $\omega$ can be obtained from Eq. (8) as,

$$
\begin{aligned}
\rho_{\mathrm{pairs}} & =\int_{0}^{\infty} d \omega \tilde{\chi}_{\gamma_{1} \gamma_{2}}(\mathbf{q} \sim 0, \omega) \\
& \approx 2 \pi Z_{F}^{2} \frac{1}{N} \sum_{\mathbf{k}} \gamma_{1 \mathbf{k}} \gamma_{2 \mathbf{k}} \frac{\bar{\Delta}_{Z}^{2}(\mathbf{k})}{E_{\mathbf{k}}^{2}} \tanh \left[\frac{1}{2} \beta E_{\mathbf{k}}\right]
\end{aligned}
$$

However, the sum $\sum_{\mathbf{k}}\left(\bar{\Delta}_{Z}^{2}(\mathbf{k}) / E_{\mathbf{k}}^{2}\right) \tanh \left(\beta E_{\mathbf{k}} / 2\right)$ is equal to $4 \sum_{\mathbf{k}}\left(U_{\mathbf{k}} V_{\mathbf{k}}\right)^{2} \tanh \left(\beta E_{\mathbf{k}} / 2\right)$, where the electron quasiparticle coherence factors $U_{\mathbf{k}}^{2} \approx U_{2 h \mathbf{k}+\mathbf{k}_{\mathbf{A}}}^{2}$ and $V_{\mathbf{k}}^{2} \approx$ $U_{1 h \mathbf{k}+\mathbf{k}_{\mathbf{A}}}^{2}$ describe the probabilities of the Cooper pair being occupied and unoccupied, respectively. Furthermore, this sum is not vanishing only around the Fermi energy in the range of $2 \bar{\Delta}_{Z}(\mathbf{k})$. This quantity corresponds to DOCP 22], formed around the Fermi surface as the energy gap is opening. In this sense, $\rho_{\text {pairs }}$ is proportional to DOCP, weighted by the Raman vertex [22]. In this case, we have performed a calculation for the doping dependence of $\rho_{\text {pairs }}$, and the results of (a) $\rho_{\text {pairs }}^{\mathrm{B}_{1 g}}$ (solid line) and $\rho_{\text {pairs }}^{\mathrm{B}_{2 g}}$ (dashed line) and (b) the sum of $\rho_{\text {pairs }}^{\mathrm{B}_{1 g}}$ and $\rho_{\text {pairs }}^{\mathrm{B}_{2 \mathrm{~g}}}$ as a function of doping with $T=0.002 J$ are plotted in Fig. 5 in comparison with the corresponding experimental data 22] (inset). The present results show that in the momentum space, $\rho_{\text {pairs }}$ is strongly anisotropic as a function of doping. Although $\rho_{\text {pairs }}^{\mathrm{B}_{1 \mathrm{~g}}}$ is much larger than $\rho_{\text {pairs }}^{\mathrm{B}_{2 g}}$ in the corresponding doping, they almost linearly increase with increasing doping in the underdoped regime. In particular, at low doping, $\rho_{\text {pairs }}^{\mathrm{B}_{1 g}}$ and $\rho_{\text {pairs }}^{\mathrm{B}_{2 g}}$ become very small and vanishe below $p \sim 0.05$. Moreover, in a similar way to the superfluid density, the sum of $\rho_{\text {pairs }}^{\mathrm{B}_{1 \mathrm{~g}}}$ and $\rho_{\text {pairs }}^{\mathrm{B}_{2 g}}$, which describes the overall DOCP, increases linearly with increasing doping in the underdoped regime, in qualitative agreement with the experimental data [22].

A nature question is why the doping and temperature dependence of the $B_{1 g}$ spectrum in the overdoped regime and $B_{2 g}$ spectrum throughout the $\mathrm{SC}$ dome in cuprate superconductors can be described qualitatively within the $\mathrm{KE}$ driven $\mathrm{SC}$ mechanism. The reason is that the $\mathrm{KE}$ driven SC state is the conventional d-wave BCS like 24, 27]. In particular, this $\mathrm{KE}$ driven d-wave $\mathrm{SC}$ state is controlled by both SC gap and quasiparticle coherence, then the maximal $T_{c}$ (then gap parameter) occurs around the optimal doping, and decreases in both underdoped and overdoped regimes. On the other hand, based on the phenomenological BCS formalism with a simple d-wave gap $\Delta_{\mathbf{k}}=\Delta\left[\cos k_{x}-\cos k_{y}\right]$, it has been shown [6] that the positions of the maxima in the $B_{1 g}$ and $B_{2 g}$ scattering both scale with the gap parameter $\Delta$. However, in the present paper, the ERR function of cuprate superconductors (5) is obtained in terms of the imaginary part of RDDCF (8) within the KE driven SC mechanism, although its expression is similar to that obtained within the phenomenological d-wave BCS formalism [6]. This is why the doping and temperature dependence of the $B_{1 g}$ spectrum in the overdoped regime and $B_{2 g}$ spectrum throughout the SC dome in cuprate superconductors can be described qualitatively within the KE driven $\mathrm{SC}$ mechanism.

Although many different theories have been put forward in order to explain the nature of ERR in the $B_{1 g}$ orientation in the underdoped regime, however, its full understanding is still controversial. One point of view is that the experimental result from ERR [10 15] does not necessary imply two distinct gaps, but still it does imply that the gap behaves differently in the nodal and antinodal momentum regimes. In particular, it has been argued that the ERR data in both $B_{1 g}$ and $B_{2 g}$ geometries 
in the underdoped regime can be equally well explained within a one-gap scenario if final-state interactions are taken into account 28]. In other theories, the normal state pseudogap is associated with the nature of the $B_{1 g}$ peak energy in the underdoped regime 29]. However, in the present case, the continuation of the treatments from the SC to normal states is not straightforward. This can be seen directly from Eq. (9), the intensity of ERR vanishes proportional to $\bar{\Delta}_{Z}^{2}(\mathbf{k})$ as $T \rightarrow T_{c}$. In this case, ERR in the underdoped regime has been studied within the fluctuation-exchange approximation for the Hubbard model by phenomenologically introducing a pseudogap [30], where this pseudogap leads to that a corresponding peak evolves continuously in the $B_{1 g}$ spectrum as $T$ decreases in the normal state and below $T_{c}$. However, this theory can not be applied to discuss the doping dependent behaviors of ERR in $B_{1 g}$ and $B_{2 g}$ symmetries.

Within the KE driven SC mechanism, our present result of the doping and temperature dependence of ERR in the $B_{1 g}$ symmetry is in disagreement with the experimental data $7-16,22]$ in the underdoped regime. In Ref. 14, it has been argued that for a correct description of the $B_{1 g}$ spectrum in the underdoped regime, two essential ingredients should be taken into account within the d-wave BCS formalism: (a) the quasiparticle spectral weight $Z_{F}(\mathbf{k})$ as well as the vertex correction; and (b) a general gap also should be taken into account by including the higher order of the harmonic component in the simple d-wave gap function $\Delta_{\mathbf{k}}=\Delta\left[\cos k_{x}-\cos k_{y}\right]$. In our present studies, the first condition is partially satisfied, since the doping dependence of the quasiparticle spectral weight $Z_{F}$ has been included in our above discussions. However, the vertex correction for the interaction of the quasiparticles due to the presence of the spin fluctuation is not included, which also is doping and momentum dependent. For the second condition, a more general gap $\Delta_{\mathbf{k}}=\Delta\left[\cos k_{x}-\cos k_{y}+B\left(\cos 2 k_{x}-\cos 2 k_{y}\right)\right]$ has been observed [31]. Although this gap function still is basically consistent with the d-wave symmetry, it is obviously that there is a significant deviation from the simple d-wave form. On the other hand, the final-state interactions are also very important for interpreting the unusual $B_{1 g}$ spectrum in the underdoped regime as point out in Ref. 28, and should be taken into account. Thus an important issue is how these essential ingredients that have been dropped in the present studies are taken into account within the KE driven SC mechanism for a correct description of the $B_{1 g}$ spectrum in the underdoped regime. These and the related issues are under investigation now.

In summary, we have studied the doping and temperature dependence of ERR in cuprate superconductors based on the KE driven SC mechanism. Our results show that the temperature dependent depletion at low-energy shifts is faster in the $B_{1 g}$ symmetry than in the $B_{2 g}$ symmetry. When increasing doping, the intensities of the peaks in both $B_{1 g}$ and $B_{2 g}$ orientations are strongly increased. In particular, in analogy to the domelike shape of the doping dependence of $T_{c}$, the maximal peak energy in the $B_{2 g}$ channel occurs around the optimal doping, and then decreases in both underdoped and overdoped regimes. Moreover, in a similar way to the superfluid density, the overall DOCP increases with increasing doping in the underdoped regime.

\section{Acknowledgments}

This work was supported by the National Natural Science Foundation of China under Grant Nos. 10774015 and 11074023, and the funds from the Ministry of Science and Technology of China under Grant Nos. 2006CB601002 and 2006CB921300.
[1] See, e.g., the review, A. Damascelli et al., Rev. Mod. Phys. 75 (2003) 473.

[2] See, e.g., the review, C. C. Tsuei and J. R. Kirtley, Rev. Mod. Phys. 72 (2000) 969.

[3] J. R. Schrieffer, Theory of Superconductivity, Benjamin, New York, 1964.

[4] P. W. Anderson, Science 235 (1987) 1196.

[5] See, e.g., C. Thomsen and M. Cardona, in Physical Properties of High Temperature Superconductors I, edited by D. M. Ginsberg (World Scientific, Singapore, 1989).

[6] See, e.g., the review, T. P. Devereaux and R. Hackl, Rev. Mod. Phys. 79 (2007) 175.

[7] T. Staufer et al., Phys. Rev. Lett 68 (1992) 1069.

[8] X. K. Chen et al., Phys. Rev. Lett 73 (1994) 3290.

[9] X. K. Chen et al., Physica C 295 (1998) 80.

[10] M. Opel et al., Phys. Rev. B 61 (2000) 9752.

[11] K. C. Hewitt and J. C. Irwin, Phys. Rev. B 66 (2002) 054516.

[12] F. Venturini et al., J. Phys. Chem. Solids 63 (2002) 2345.
[13] Y. Gallais et al., Phys. Rev. B 71 (2005) 012506.

[14] M. LE Tacon et al., Nature Physics. 2 (2006) 537.

[15] W. Guyard et al., Phys. Rev. B 77 (2008) 024524.

[16] W. Guyaid et al., Phys. Rev. Lett 101 (2008) 097003.

[17] T. P. Devereaux et al., Phys. Rev. Lett. 72 (1994) 396; D. Branch and J. P. Carbotte, Phys. Rev. B 54 (1996) 13288.

[18] D. Manske et al., Phys. Rev. B 56 (1997) R2940.

[19] D. Branch and J. P. Carbotte, Phys. Rev. B 52 (1995) 603.

[20] T. Dahm et al., Phys. Rev. B 58 (1998) 12454.

[21] A. V. Chubukov et al., Phys. Rev. B 73 (2006) 094512.

[22] S. Blanc et al., Phys. Rev. B 80 (2009) 140502(R).

[23] Y. J. Uemura et al., Phys. Rev. Lett. 62 (1989) 2317.

[24] Shiping Feng, Phys. Rev. B 68 (2003) 184501; Shiping Feng et al., Physica C 436 (2006) 14.

[25] Shiping Feng et al., J. Phys. Condens. Matter 16 (2004) 343.

[26] See, e.g., the review, Shiping Feng et al., Int. J. Mod. 
Phys. B 22 (2008) 3757.

[27] Huaiming Guo and Shiping Feng, Phys. Lett. A 361 (2007) 382 .

[28] A. V. Chubukov and M. R. Norman, Phys. Rev. B 77 (2008) 214529.
[29] See, e.g., the review, S. Hüfner et al., Rep. Prog. Phys. 71 (2008) 062501.

[30] T. Dahm et al., Phys. Rev. B 59 (1999) 14740.

[31] J. Mesot et al., Phys. Rev. Lett. 83 (1999) 840. 